\title{
Experimental study of dense snow avalanches: velocity profiles in steady and fully developed flows
}

\author{
Alexi BOUCHET, Mohamed NAAIM, Hervé BELLOT, Frédéric OUSSET \\ UR ETNA, Cemagref, Domaine universitaire, 2 rue de la Papeterie, BP 76, 38402 Saint-Martin-d'Hères Cedex, France \\ E-mail: alexi.bouchet@grenoble.cemagref.fr
}

\begin{abstract}
In order to study channelled snow flows over rough surfaces, a laboratory-scale experiment was installed at the "Col du Lac Blanc", a $2800 \mathrm{~m}$ high pass in the French Alps, near the Alpe d'Huez ski resort. It consists of a $0.2 \mathrm{~m}$ wide, $10 \mathrm{~m}$ long channel fed with snow by a motorized hopper. Both the slope of the channel and the feeding rate of the hopper can be modified. Sensors in the channel provide measurements of the velocity profile, the flow height and the shear and normal stresses at the bottom of the flow. Velocity profiles for different slopes are presented in this paper. Results indicate the presence of a highly active layer at the bottom. This layer is mainly responsible for the avalanche velocity, while the upper layer has a much smaller velocity gradient. A first interpretation of both layers is given.
\end{abstract}

\section{INTRODUGTION}

Many models have been proposed to describe dry-snow avalanches (see, e.g., Salm, 1966; Perla and others, 1980; Dent and Lang, 1983; Hutter, 1996), but very few data are available to calibrate the parameters of these models. It is consequently a great challenge to obtain these parameters by means of measurements on dry-snow flows.

The question of velocity profiles in snow flows has already been investigated by Nishimura and Maeno (1989) and Tiefenbacher and Kern (2004) in a mini-avalanche system and Dent and others (1998) in a real avalanche. They all observed a thin, highly sheared layer at the bottom, with a large upper layer whose velocity gradient was much smaller (even rigid in the case of Nishimura and Maeno's measurements). To our knowledge, no systematic studies of velocity profiles for different slopes and height have been performed. For this reason, we built a system to produce snow flows at various slopes and rates. Our purpose was to produce steady-state flows, so the region in which the flow occurs had to be long enough. This enables us to measure velocity profiles of "dry snow flow" for slopes in the range $31-39^{\circ}$ which are presented in this paper.

Typical values of height and velocities for the flows we observed are $h \approx 80 \mathrm{~mm}$ and $v \approx 4 \mathrm{~m} \mathrm{~s}^{-1}$. The approximate Froude number is thus $\mathrm{Fr} \approx 4.5$. Such flows are more than one order of magnitude smaller than real avalanches, and the extrapolation to a larger scale is hypothetic.

\section{SET-UP}

\subsection{The device}

The experimental set-up we built, its performance and the experimental procedure are fully described in Bouchet and others (2003). The set-up is shown in Figure 1. It consists of an inclined channel with an adjustable slope. The snow is fed by a motorized hopper. The channel is clamped to a beam whose inclination is adjustable between $27^{\circ}$ and $41^{\circ}$ in increments of $2^{\circ}$. As section 3 will establish, steady-state flows were observed in part of this range. The channel is

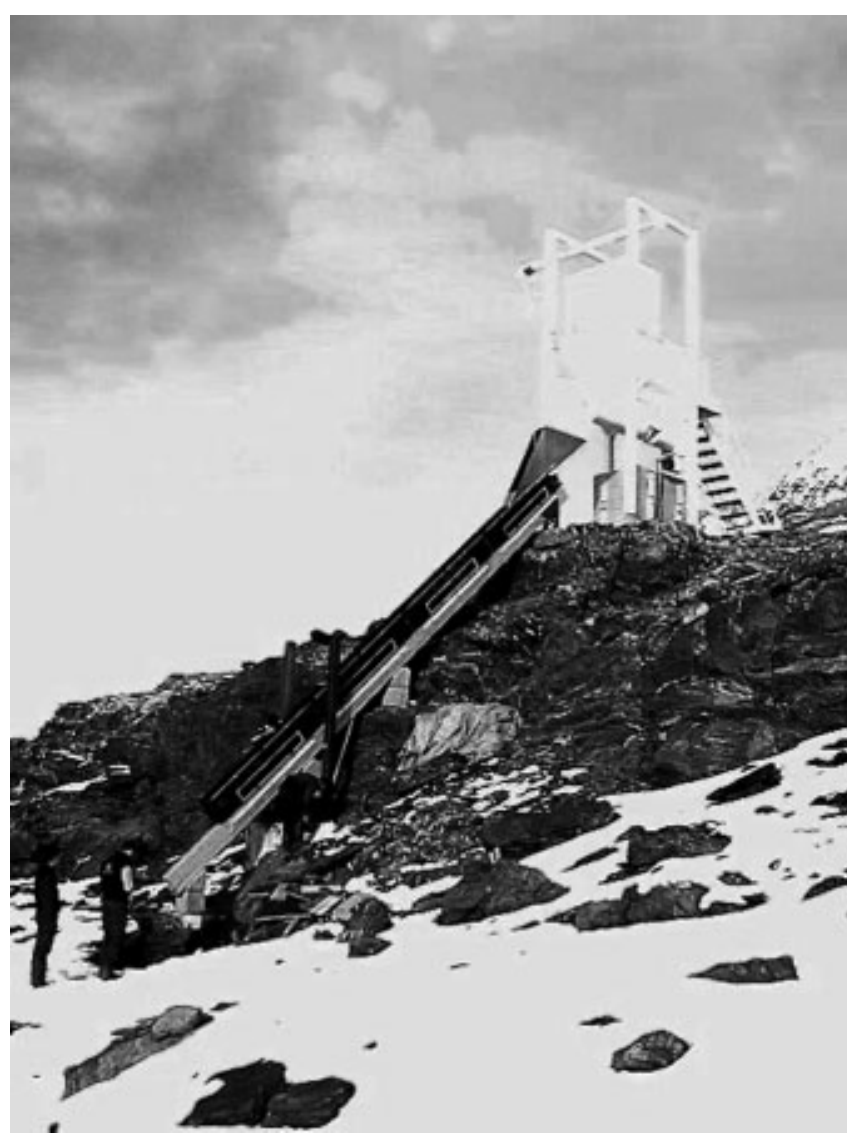

Fig. 1. The experimental set-up at the "Col du Lac Blanc", Alpe d'Huez, French Alps (2800 m a.s.l.). 

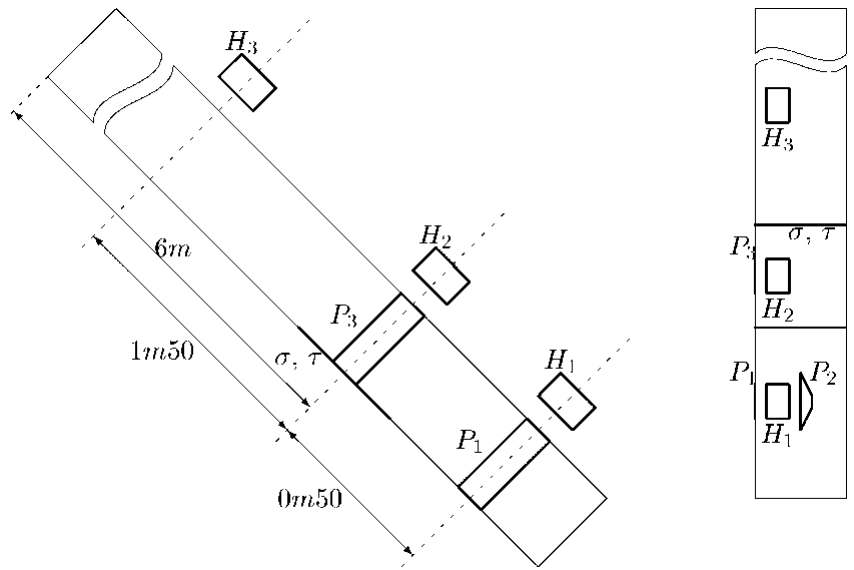

Fig. 2. Sketches of the sensor positions along the channel. The left one is a view from the side, and the right one is a view from the top.

$10 \mathrm{~m}$ long, $0.2 \mathrm{~m}$ wide and $0.2 \mathrm{~m}$ high. The feeding system consists of a storage hopper with an Archimedean screw underneath to drag the snow from the hopper into the channel at a constant rate. The screw has a slightly increasing thread lead to prevent compaction of the snow during the experiment. It is set in motion by a diesel engine linked to the screw by a hydraulic engine. This enables us to set a constant rate at any value $<220 \mathrm{~m}^{3} \mathrm{~h}^{-1}$. Since the storage capacity of the hopper is around $5 \mathrm{~m}^{3}$, the flow can last from one to several minutes. However, to prevent too much compaction of the snow, the hopper is filled only to one-third of its capacity, leading to flows of about 20-30 s. Since the channel is narrower than the screw, a funnel-shaped slide links the end of the screw to the beginning of the channel. The bottom of the channel has been covered with sandpaper to create a rough bottom and hence a shear layer of significant depth. Underneath, it has a double bottom where the electronic systems required for the sensors are installed. The flow is released by starting the screw and reaches steady state after approximately $1 \mathrm{~s}$.

\subsection{The instrumentation}

The sensors installed in the channel (sketched in Fig. 2) provide measurements of:

the normal $(\sigma)$ and shear $(\tau)$ stresses at the bottom of the channel, $6 \mathrm{~m}$ downstream from the top of the channel, using piezoelectric sensors;

the flow height at three different locations $-\mathrm{H}_{1}, 4.5 \mathrm{~m}$ downstream from the top of the channel, $\mathrm{H}_{2}, 6$ m down-

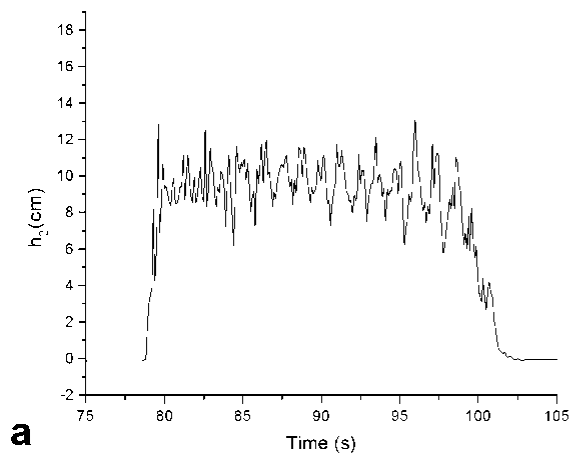

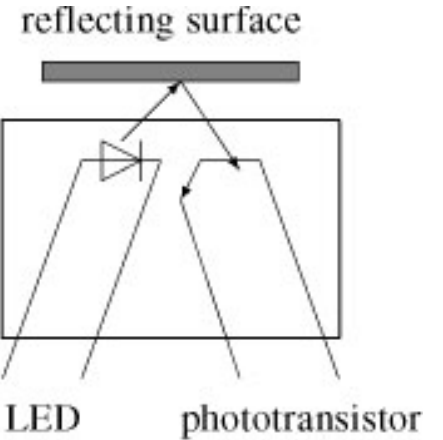

Fig. 3. Schematic drawing of the optical devices used in the velocity sensors.

stream (above the stress measurements) and $\mathrm{H}_{3}, 6.5 \mathrm{~m}$ downstream - using optical distance sensors above the flow;

the velocity profiles at three different locations: $6 \mathrm{~m}$ downstream from the top of the channel, on the sidewall (profile $\mathrm{P}_{3}$, below the measure of $\mathrm{H}_{2}$ ), and $6.5 \mathrm{~m}$ downstream, both on the side-wall (profile $\mathrm{P}_{1}$ ) and in the middle of the channel (profile $\mathrm{P}_{2}$ ).

For the velocity measurement, we used the method developed by Dent and others (1998). This is based on the correlation of two signals obtained by two identical sensors which are displaced from each other in the flow direction. Each velocity sensor is made of two identical reflectivity sensors which consist of an infrared light-emitting diode (LED) and a phototransistor as represented in Figure 3. These devices are very sensitive to the amount of light collected by the phototransistor. Since this amount of light depends on the granularity state of the reflecting surface, the signal obtained is characteristic of the snowpack which reflected the light from the LED to the phototransistor.

When the velocity direction is known, two such devices displaced at a distance $d$ in the velocity direction from one another, will give identical signals with a time shift $\delta t=d / v$, where $v$ is the velocity. To improve the performance of this method, we used three devices instead of two. The first and second devices are separated by a distance in the flow direction $d_{1}=30 \mathrm{~mm}$, and the second and third by a distance $d_{2}=20 \mathrm{~mm}$. Each velocity measurement is thus performed three times with distances between the two devices of 20,30 and $50 \mathrm{~mm}$. The velocity profile is then determined by using nine of these sensors placed in a flownormal line from the bottom with a spacing of $10 \mathrm{~mm}$. They are separated from the flow by a $3 \mathrm{~mm}$ thick glass window.

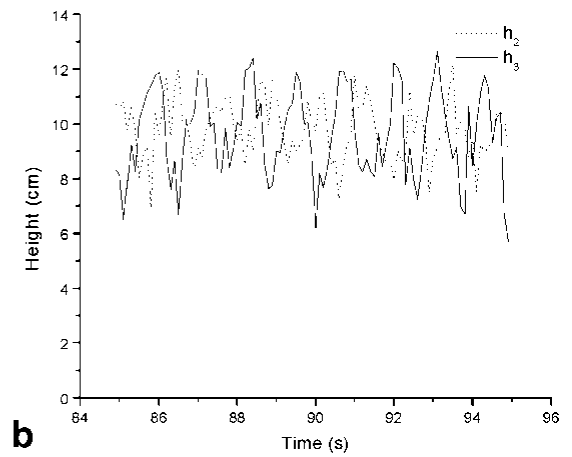



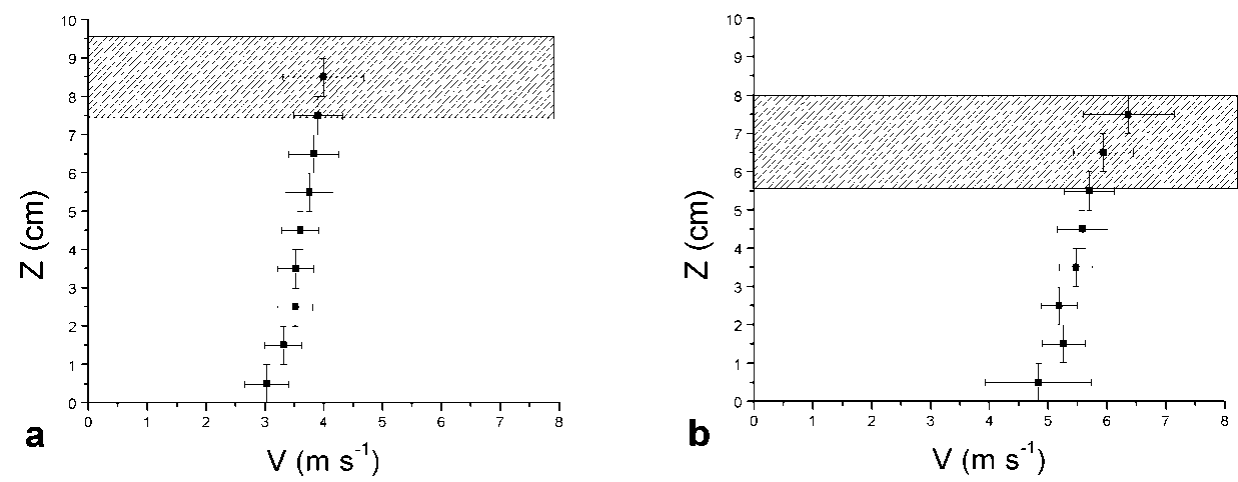

Fig. 5. Two examples for different slopes and heights of the time-averaged velocity profiles. The hatched box on top represents the minimum and maximum height measurements during the flow duration. The slope angles for these profiles were $31^{\circ}$ for $(a)$ and $39^{\circ}$ for $(b)$.

All the results presented below were obtained with flows of small rounded snow grains. The densities varied from 220 to $360 \mathrm{~kg} \mathrm{~m}^{-3}$.

\section{FLOW-HEIGHT MEASUREMENTS}

Typical variations of the heights $h_{2}$ and $h_{3}$ during the flow are presented in Figure $4 \mathrm{a}$ and b. From these graphs, two observations can be made.

1. The height has periodic variations during the flow (Fig. 4a). We observed that the maxima of these variations travelled with the flow. Our interpretation is that these variations are not waves intrinsic to the flow but are due to the feeding system.

2. The variations of $h_{2}$ follow those of $h_{3}$ (Fig. 4b). In a first approximation, it can be written that: $h_{2}(t) \approx$ $h_{3}(t-\delta t)$, where $\delta t$ is a delay approximately equal to $0.5 \mathrm{~s}$.

Considering the first point, one cannot rigorously say that steady-state conditions are achieved. Still, if $T$ is the period of the height temporal variations, then the average of the flow height over time $T$ at the instant $t$, written $\left\langle h_{2}\right\rangle_{T}(t)$, has almost no dependence on $t$ :

$$
\begin{aligned}
\left\langle h_{2}\right\rangle_{T}(t) & =\frac{1}{T} \int_{t}^{t+T} h_{2}\left(t^{\prime}\right) \mathrm{d} t^{\prime} \\
\frac{\partial\left\langle h_{2}\right\rangle_{T}}{\partial t} & \approx 0 .
\end{aligned}
$$

Such a flow can be described as steady. No regular increase or decrease of the height can be observed during the flow.

From the second point, we can say that when averaged over the period $T$, the heights $\left\langle h_{2}\right\rangle_{T}(t)$ and $\left\langle h_{3}\right\rangle_{T}(t)$ are equal. This implies that the flow has reached its terminal velocity and is fully developed:

$$
\frac{\partial\langle h\rangle_{T}(t)}{\partial x} \approx 0 .
$$

Thus, when averaged over the flow duration, the measured quantities will be the same as if the flow had reached steady state.

\section{VELOGITY PROFILES}

In this section, only the measurements obtained from velocity profile $\mathrm{P}_{3}$ (the upstream one; see Fig. 2) are pre- sented. The principle of the sensors and the amount of signal processing required to go from the raw signals to the velocity makes it very rare to obtain an instantaneous velocity profile containing all the sensors (i.e having all the sensors giving a "reasonable" result). Therefore, based on the fact mentioned above that height variations are periodic, it is more accurate to present the velocity profiles obtained when averaging the measurements over the flow duration.

Two examples of the profiles obtained are presented in Figure $5 \mathrm{a}$ and b. Figure $5 \mathrm{a}$ corresponds to flow No. 31 in Table 1. It was obtained with a slope angle of $31^{\circ}$, a snow density of $220 \mathrm{~kg} \mathrm{~m}^{-3}$ and a temperature around $-25^{\circ} \mathrm{C}$ (for both snow and air). Figure $5 \mathrm{~b}$ corresponds to flow No. 73 in Table 1. It was obtained with a slope angle of $39^{\circ}$, a snow density of $270 \mathrm{~kg} \mathrm{~m}^{-3}$ and a temperature around $-20^{\circ} \mathrm{C}$ (for both snow and air). On these profiles, the horizontal error bars are the standard deviation of the velocity distribution for each sensor during the flow, and the vertical ones are the flow-normal dimension of the sensors. On both profiles, one can notice that the velocity of the lower sensor is non-zero. This means either that there is a highly sheared thin layer at the bottom (the sensor is $1 \mathrm{~cm}$ high and is placed at the bottom of the channel, so the shear rate would at least be greater than $\dot{\gamma}_{\text {min }} \simeq 500 \mathrm{~s}^{-1}$ ) or that the snow is slipping on the bottom. Since we have no way of observing a layer thinner than $10 \mathrm{~mm}$ with our sensors, we chose to describe this layer by a slip condition. Above this slip layer, the

Table 1. Characteristics of the flows

\begin{tabular}{cccccc}
\hline Flow No. & Slope & Height & Density \\
& & $\mathrm{mm}$ & $V_{\mathrm{s}}$ & $\dot{\gamma}$ \\
& & & & & $\mathrm{kg} \mathrm{m}^{-3}$ \\
$\mathrm{~m} \mathrm{~s}^{-1}$ & & \\
\hline & & & & & \\
31 & $31^{\circ}$ & 85 & 220 & 3.12 & 10.8 \\
41 & $31^{\circ}$ & 73 & 220 & 3.03 & 13.55 \\
42 & $33^{\circ}$ & 74 & 360 & 3.08 & 15.3 \\
52 & $33^{\circ}$ & 83.5 & 360 & 3.20 & 12.5 \\
53 & $35^{\circ}$ & 82.5 & 220 & 4.28 & 11.1 \\
60 & $35^{\circ}$ & 76 & 220 & 3.61 & 10.6 \\
63 & $37^{\circ}$ & 80 & 250 & 4.24 & 13.1 \\
71 & $37^{\circ}$ & 95.5 & 250 & 3.99 & 9.24 \\
72 & $39^{\circ}$ & 62.5 & 270 & 4.88 & 14.4 \\
73 & $39^{\circ}$ & 60.5 & 270 & 4.94 & 13.5 \\
74 & $39^{\circ}$ & 68 & 270 & 4.95 & 14.4 \\
& $39^{\circ}$ & 93.5 & 270 & 4.75 & 13.5 \\
\hline
\end{tabular}

*Averaged over the flow duration.

${ }^{\dagger}$ Measured in the hopper just below the flow. 

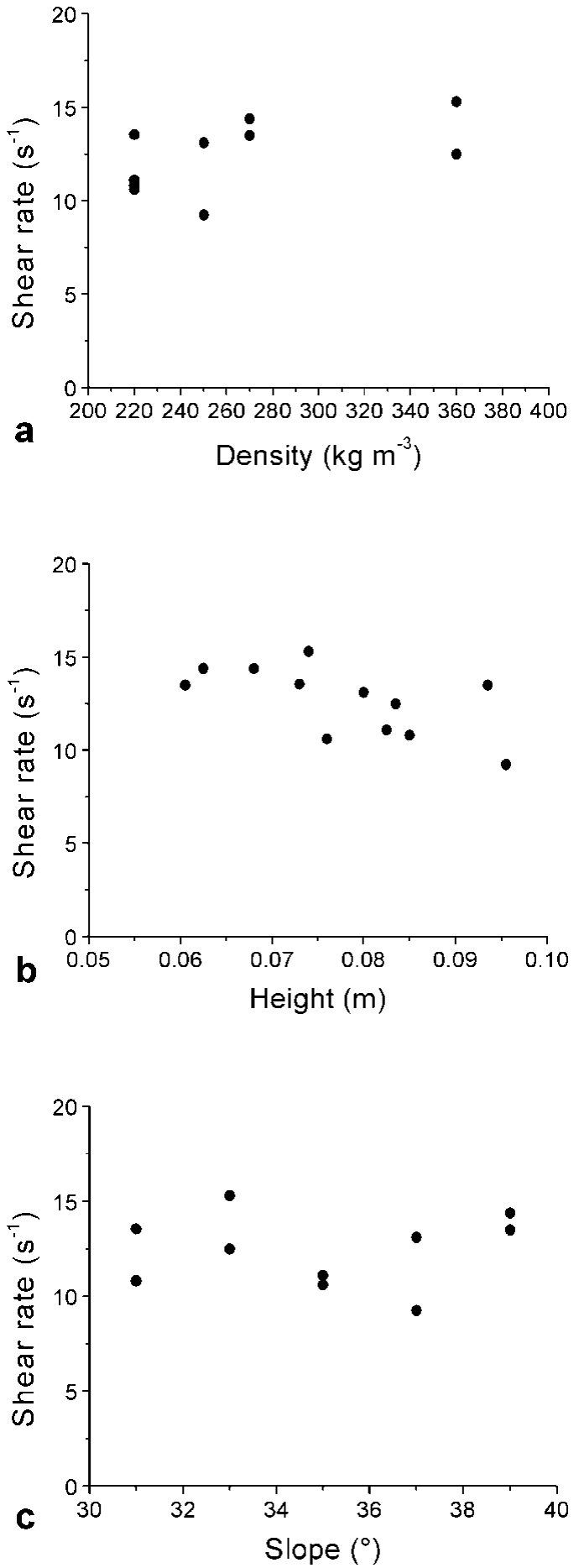

Fig. 6. Variations of the shear rate $\dot{\gamma}$ with density (a), height (b) and slope (c).

velocity increases slightly but regularly, with an almost uniform velocity gradient. Since both these characteristics were observed for every flow, we performed a linear fit for each profile with the function:

$$
V(z)=V_{\mathrm{s}}+\dot{\gamma} z
$$

Each profile is thus described by two parameters:

1. A slip velocity $V_{\mathrm{s}}$

2. A uniform shear rate $\dot{\gamma}$

The characteristics (slope, height and density) of the flows and the results obtained are presented in Table 1.

The shear rates obtained are very similar to each other.

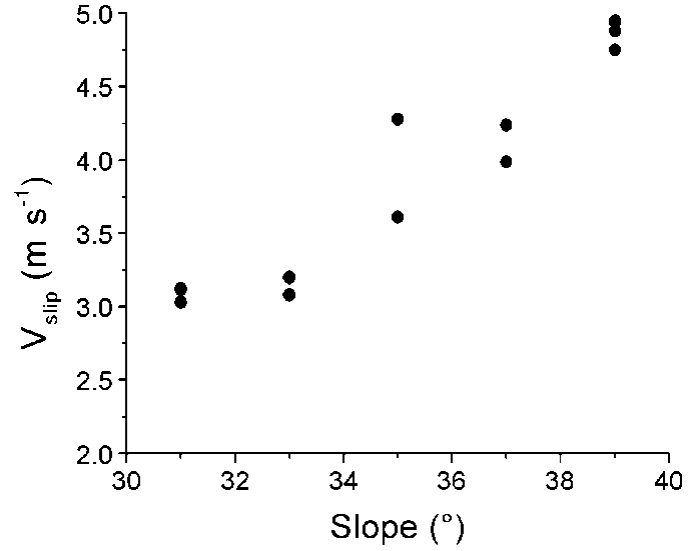

Fig. 7. Dependence of the slip velocity on the slope.

Besides, as seen in Figure 6a and b, no specific dependences upon the slope, height or density can be observed in the range investigated. The slip velocity $V_{\mathrm{s}}$, however, increases with the slope as represented in Figure 7.

\section{COMPARISON WITH OTHER RESULTS}

In this section, the two characteristics of our velocity profiles (a uniform velocity gradient and a slip velocity at the bottom) are compared to previous measurements of snow properties.

\subsection{Velocity profiles in snow flows or avalanches}

Velocity profiles have been measured in snow flows by Nishimura and Maeno (1989) and Tiefenbacher and Kern (2004) and in real avalanches by Dent and others (1998). The dimensions of their experiments and the conditions in which they were performed are fairly different. It is thus not surprising that they lead to different results.

A device similar to ours was used by Nishimura and Maeno (1989). It consists of a $0.1 \mathrm{~m}$ wide, $6 \mathrm{~m}$ long channel. The snow was fluidized by air injection before its release, and the flow lasted approximately $3 \mathrm{~s}$. The velocity profiles obtained were interpreted as those of a threshold fluid: an upper layer in which particles move as a rigid body, and a sheared layer underneath with a parabolic profile. The sheared layer is $20-30 \mathrm{~mm}$ thick.

Dent and others (1998) performed their measurements in a real avalanche. The profiles had a layer thinner than $10 \mathrm{~mm}$ at their bottom, where the velocities increase to $3-4 \mathrm{~m} \mathrm{~s}^{-1}$. On top of this layer, the shear rate drops to a value at least an order of magnitude smaller. The thin layer at the bottom is interpreted as being mainly responsible for the speed of the avalanche.

The "Weissfluhjoch chute" was used by Tiefenbacher and Kern (2004). It consists of a $34 \mathrm{~m}$ long, $2.5 \mathrm{~m}$ wide channel. The velocity profiles obtained are similar in shape to those obtained by Nishimura and Maeno (1989), with a high sheared layer at the bottom, approximately $50 \mathrm{~mm}$ thick, and a quasi-uniform "plug-flow" layer above. The sheared part of the flow was approximated by a parabolic profile.

Our profiles differ from those obtained by Nishimura and Maeno (1989) and Tiefenbacher and Kern (2004), but the 


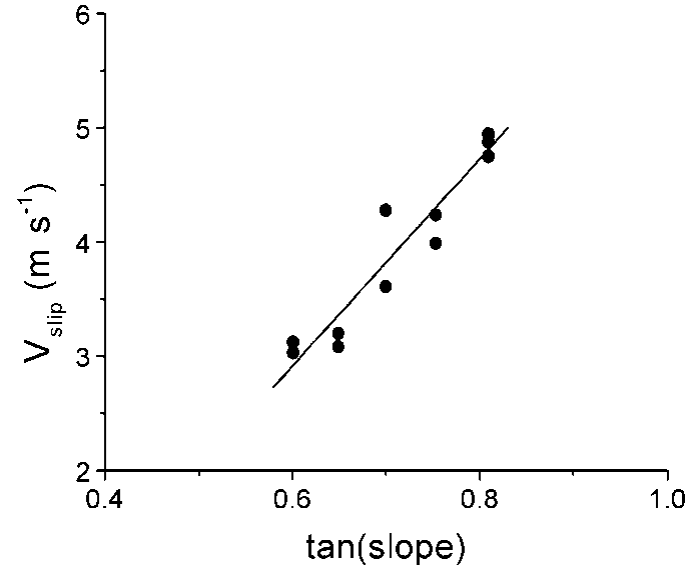

Fig. 8. Linear fit of the dependence of the slip velocity on the slope: $V_{\mathrm{s}}=-V_{\text {offset }}+V_{0} \tan \theta$. Parameters are: $V_{\text {offset }}=$ $2.53 \mathrm{~m} \mathrm{~s}^{-1}$ and $V_{0}=9.08 \mathrm{~m} \mathrm{~s}^{-1}$.

flow regime they observed also differs from ours: we study a quasi-steady-state flow, while theirs are in a transitory state. The profiles we measured are similar to those observed in a real avalanche: a thin $(<10 \mathrm{~mm}$ thick), highly sheared layer at the bottom, surmounted by an upper layer with an approximately uniform shear rate. This constant shear rate in the upper layer disproves the existence of a simple relationship $\dot{\gamma}=\Gamma(\tau)$ between the shear rate $\dot{\gamma}$ and the shear stress $\tau$.

\subsection{Snow-snow friction}

As explained in section 3, flows can be considered as fully developed and steady so that quantities may be averaged over the flow duration. Therefore, the effective friction coefficient, defined as $\mu_{\text {eff }}=\tau / \sigma$ where $\tau$ and $\sigma$ are respectively the shear and normal stresses at the bottom of the flow, balances the gravity: $\mu_{\text {eff }}=\tan \theta$. Now, as represented in Figure $8, V_{\mathrm{s}}$ increases linearly with $\tan \theta$ in the range investigated: $V_{\mathrm{s}}=-V_{\text {offset }}+V_{0} \tan \theta$. Thus, the friction coefficient increases linearly with the slip velocity in the range investigated:

$$
\mu_{\mathrm{eff}}=\mu_{0}+\frac{V_{\mathrm{s}}}{V_{0}}
$$

with $\mu_{0}=0.28$ and $V_{0}=9.1 \mathrm{~m} \mathrm{~s}^{-1}$.

Similar results were reported by Casassa and others (1991) for friction measurements between two rigid snow layers. They obtained similar values of $\mu_{0}$, depending on the temperature, but $V_{0}$ in our case is five times smaller than theirs. Thus, in our experiments, the friction coefficient increases five times faster with the slip velocity than in Casassa and others' experiments. This is certainly due to the difference in roughness state between the sandpaper we use and the snow.

\section{FLOW-RATE-FLOW-HEIGHT RELATIONSHIP}

Taking into account Equations (1) and (2), the velocity within the flow can finally be written:

$$
V(z)=V_{0}\left(\tan \theta-\tan \theta_{0}\right)+\dot{\gamma} z,
$$

with $\tan \theta_{0}=\mu_{0}$. Averaging this equation over the flow height $h$ leads to the relationship between the average velocity $\bar{u}$ of the flow and the flow height in the range of slopes and height investigated:

$$
\bar{u}=V_{0}\left(\tan \theta-\tan \theta_{0}\right)+\frac{1}{2} \dot{\gamma} h
$$

with $V_{0} \approx 9.1 \mathrm{~m} \mathrm{~s}^{-1}, \quad \tan \theta_{0}=0.28 \quad\left(\theta_{0} \approx 15.5^{\circ}\right) \quad$ and $\dot{\gamma} \approx 15 \mathrm{~s}^{-1}$.

\section{GONGLUSION}

The experimental set-up installed at the "Col du Lac Blanc" enabled us to generate fully developed flows with snow with small rounded grains. Slope angles of $31-39^{\circ}$ were investigated. The observed flows were steady and fully developed. Consequently, the velocity profiles were averaged over the flow duration and analyzed as those of a steady-state flow. The profiles found are similar to those observed in a real avalanche by Dent and others (1998): a uniform shear rate within the flow and an active thin layer at the bottom, interpreted as a slip velocity. The dependence of the slip velocity on the slope angle can be interpreted in terms of a friction coefficient, and leads to results similar to snow-snow friction measurements done by Casassa and others (1991). Finally, from the shape of the profile, a flow law was derived for the range of slopes and heights investigated.

\section{AGKNOWLEDGEMENTS}

Many people have participated in the installation of the experimental set-up at the "Col du Lac Blanc" and to the huge amount of work necessary to establish a satisfactory experimental procedure. Mentioning all of them would add a couple of pages to this paper, so we would like to thank every person who helped us dig metres-deep trenches in packed snow or make reinforced concrete foundations for the set-up at the bottom of the trench. Among them, special thanks to R. Ducret, who put amazing enthusiasm into all these activities, and to D. Cauvard who did much of the dirty job of signal processing.

\section{REFERENCES}

Bouchet, A., M. Naaim, F. Ousset, H. Bellot and D. Cauvard. 2003. Experimental determination of constitutive equations for dense and dry avalanches: presentation of the set-up and first results. Surv. Geophys., 24(5-6), 555-568.

Casassa, G., H. Narita and N. Maeno. 1991. Shear cell experiments of snow and ice friction. f. Appl. Phys., 69(9), 3745-3756.

Dent, J. D., K. J. Burrell, D. S. Schmidt, M.Y. Louge, E. E. Adams and T. G. Jazbutis. 1998. Density, velocity and friction measurements in a dry-snow avalanche. Ann. Glaciol., 26, 247-252.

Dent, J. D. and T. E. Lang. 1983. A biviscous modified Bingham model of snow avalanche motion. Ann. Glaciol., 4, 42-46.

Hutter, K. 1996. Avalanche dynamics. In Singh, V. P., ed. Hydrology of disasters. Dordrecht, etc., Kluwer Academic Publishers, 317-394.

Nishimura, K. and N. Maeno. 1989. Contribution of viscous forces to avalanche dynamics. Ann. Glaciol., 13, 202-206.

Perla, R., T. T. Cheng and D. M. McClung. 1980. A two-parameter model of snow-avalanche motion. f. Glaciol., 26(94), 197-207.

Salm, B. 1966. Contribution to avalanche dynamics. International Association of Scientific Hydrology Publication 69 (Symposium at Davos 1965 - Scientific Aspects of Snow and Ice Avalanches), 199-214.

Tiefenbacher, F. and M. Kern. 2004. Experimental devices to determine snow avalanche basal friction and velocity profiles. Cold Reg. Sci. Technol. 38 (1), 17-30. 